\title{
CONFOCAL LASER SCANNING ELECTRON MICROSCOPE ASSESSMENT OF DIFFERENT ANTIBIOFILM STRATEGIES
}

\author{
Ahmed M. Farghaly* and Ahmed H.Labib**
}

\begin{abstract}
Objective: The purpose of this study was to evaluate the antibiofilm efficacy of nanoherbal medicament, calcium hydroxide, Rose Bengal and sodium hypochlorite on multispecies biofilm in infected dentin section blocks. Methods: A total of one hundred dentin section blocks were used in this study. Dentin section blocks were prepared to be $(4 \times 4 \times 1 \mathrm{~mm})$ respectively. Each dentin section was underwent sterilization using autoclave at $121^{\circ} \mathrm{C}$ for 20 minutes which was confirmed using bacterial count test. Cultivation of standard strains of E. Faecalis ATCC 29212 and Staph. Epidermidis ATCC 12228 were prepared then inoculated on dentin section blocks and incubated for 3 weeks at $37^{\circ} \mathrm{C}$ to form multispecies biofilm. The specimens were grouped into 5 groups according to final irrigant and medicament: G1: Nano punica granatum (Pomegranates) herbal extract medicament, G2: Calcium hydroxide paste medicament, G3: Rose Bengal dye (RB) irrigation activated with diode laser (Photodynamic therapy), G4: Sodium hypochlorite 5.25\% irrigation, and G5: Negative Control group. The effect of tested materials was assessed using Confocal Laser Scanning Electron Microscope (CLSM) and data were statistically analyzed. Results: The highest mean percentage of dead bacteria was found in Pomegranates followed by RB followed by sodium hypochlorite 5.25\% then the calcium hydroxide paste group. The least mean percentage of dead bacteria was found in the control group. With a statistically significant difference between groups where $\mathrm{p}<0.001$. Conclusion: Nano punica granatum herbal extract medicament and Rose Bengal dye (RB) irrigation (Photodynamic therapy) could be considered potent antibiofilm strategies for disinfection of the root canal system.
\end{abstract}

\section{INTRODUCTION}

One of the most important goals of root canal treatment is to eliminate bacteria from the infected root canal systems through instrumentation and use of disinfecting agents ${ }^{(\mathbf{1 , 2})}$. Biofilm can be defined as a sessile multicellular microbial community characterized by cells that are firmly attached to a surface and enmeshed in a self-produced matrix of extracellular polymeric substance (EPS) $)^{(3,4)}$. Its elimination from anatomical complexity of the root canal system is a challenging procedure. Thus; the use of a potent antibiofilm strategy has become mandatory to be able to resist endodontic infection ${ }^{(5,6)}$.

Sodium hypochlorite in concentrations from $0.5 \%-6 \%$ is the most commonly recommended root canal irrigant. Aqueous solution of sodium hypochlorite is a dynamic balance of sodium hydroxide and hypochlorous acid, which on interaction with microorganisms and organic tissue causes chloramination, amino acid neutralization, and saponification reactions leading to strong antibacterial and tissue-dissolving effects ${ }^{(7)}$.

Calcium hydroxide is the most commonly used intracanal medication during root canal procedures $^{(8)}$. Its antibacterial property is generally related to the release of hydroxyl ions, which produces the lethal effects on bacterial cells including protein denaturation and damage to the bacterial cytoplasmic membranes and DNA ${ }^{(9)}$. However, the antimicrobial activity of sodium hypochlorite and calcium hydroxide can be inactivated by dentin, exudate from the periapical area, and microbial biomass ${ }^{(10)}$. In addition, both sodium hypochlorite and calcium hydroxide do not always eliminate $E$. faecalis biofilms from the root canal system.

\footnotetext{
* Associate Professor of Endodontic, Faculty of Dental Medicine(Boys, Cairo), Al-Azhar University

** Associate Professor of Endodontic, Faculty of Dentistry, Tanta University
} 
Recently, Photodynamic therapy (PDT) as an antibiofilm strategy is based on the use of a nontoxic dye (photosensitizer agent), that when activated by using a low energy light lead to production of free radicals such as singlet oxygen ${ }^{(11)}$. Singlet oxygen generated is highly reactive and is known to target various bacterial sites such as cell wall, nucleic acid, lipids membrane and proteins membrane, which promote bacterial cell death. Therefore, it has potentiality to enhance the disinfection efficacy of the conventional chemo-mechanical preparation ${ }^{(12)}$.

Nanoparticles are insoluble particles that are ranging from 1 to $100 \mathrm{~nm}$ in size which combines biology principles with physical and chemical procedures to generate nano-sized particles with specific functions ${ }^{(13)}$. Different nanoparticles have been introduced as an irrigant or medicament to control the bacterial biofilm in root canal system.

Pomegranates (Punica granatum L.) which have a long history of antibacterial use dating back to biblical times ${ }^{(14)}$. Egyptians used pomegranates to treat a number of different infections. It was utilized as a traditional remedy for thousands of years under the Ayurvedic system of medicine, with extracts from the rind of the fruit and bark of the tree being effective against diarrhea and dysentery. The Pomegranates in nanosized form have been applied in many health care fields because of their broadspectrum bactericidal and virucidal properties (15). So, the aim of this study was to evaluate the antibiofilm efficacy of nanoherbal medicament and photodynamic therapy using Confocal Laser Scanning Microscope (CLSM).

\section{MATERIALS AND METHODS}

1. Selection of the teeth: A total of one hundred non-carious recently extracted human single rooted teeth with fully formed root apices were collected from patients with ages ranging between 20 and 45 years old. The selected teeth were cleaned of calculus and soft tissue remnants using a hand curette. A dental operating microscope $(8 \mathrm{x})$ were used to examine the selected teeth. Also to confirm the presence of type I root canal morphology in each tooth according to Vertucci's classification. The teeth that had caries, deep cracks, attritions, fractures or restorations would be excluded from the study.

2. Preparation of the samples: The dentin section blocks were prepared and standardized to be $(4 \times 4 \times 1 \mathrm{~mm})$ (Length $\times$ Width $\times$ thickness) according to Haapasalo \& Orstavik D. ${ }^{(16)}$ technique through the following steps: - Demarcations were done at CEJ and at the last $3 \mathrm{~mm}$ of the roots. The crowns of demarcated teeth were cut off at the level of C.E.J by using a diamond disc mounted in a straight hand piece under water coolant. Root canal patency for each sample was done using a size \#10 and \#15 K file. Instrumentation was done using Revo-S rotary NiTi file system according to the manufacturer's instructions. Rotary files were mounted in a torque limited control motor at a torque $1.8 \mathrm{Ncm}$ and speed $400 \mathrm{rpm}$ as recommended by the manufacture starting from SC1(25 taper 0.06) to AS40(40 taper 0.06). Irrigation was done using side vented needle gauge 30 mounted on 3 milliliters plastic syringe two millimeters shorter than the working length using 3 milliliters of $5.25 \% \mathrm{NaOCl}$ between each instrumentation during the cleaning and shaping procedure. The apical $3 \mathrm{~mm}$ of the roots were sectioned off and the roots were longitudinally sectioned in bucco-lingual direction along the midsagittal plane into two semicylindrical halves and the cementum was removed from the root surface using Isomete saw at $1000 \mathrm{rpm}$ under water cooling. Each samples of the roots were shaped and refined to be $(4 \times 4 \times 1 \mathrm{~mm})$ (Length $\times$ Width $\times$ thickness) respectively using Isomete saw at 1000 rpm under water coolant.

3. Sterilization of the samples: Each dentin section block was placed in $1.5 \mathrm{~mL}$ Eppendorf tube filled with brain heart infusion (BHI) broth and sealed within sterilization pouches then underwent 
sterilization using autoclave at $121^{\circ} \mathrm{C}$ for 20 minutes . After completion of sterilization, the Eppendorf tubes opened in a sterile air laminar flow cabinetand a sterile paper points were inserted into the Eppendorf tubes for 1 minute until it completely saturate and absorbed the broth media, then the paper point was transferred and spread on brain heart infusion (BHI) agar plate The plate was incubated for 24 hours at $37^{\circ} \mathrm{C}$ and $100 \%$ humidity for bacterial count. After 24 hours the plate was inspected and checked for no inhibition zones around the incubated paper point indicating a sterility of the samples before inoculating the multispecies microorganisms.

\section{Preparation of the microorganisms:} Cultivation of Standard strains: Enterococcus Faecalis (E. Faecalis) American Type Culture Collection (ATCC 29212) and Staphylococcus epidermidis (Staph. epidermidis) American Type Culture Collection (ATCC 12228) were done and each Eppendorf tube contain sterile dentin section block was opened in a sterile air laminar flow cabinet. Eppendorf tubes were filled with $0.5 \mathrm{ml}$ of $E$. faecalis suspension plus $0.5 \mathrm{ml}$ of Staph.epidermidis suspension using a sterile micropipette tips for each organism. Eppendorf tubes were closed tightly and shacked well then incubated for 21 days at $37^{\circ} \mathrm{C}$ in incubator. Antagonism test was done between two microorganisms by plate-cultured diffusion method to confirm that the interaction between Enterococcus faecalis and Staphylococcus epidermidis to form multispecies biofilm was symbiotic interaction (no mutual harmful effects to each other's).

\section{Confirmation of multispecies biofilm}

formation: After incubation period, the samples were examined for confirmation of multispecies biofilm formation on dentin section blocks. Three randomly samples were selected after finishing of sterilization and before bacterial inoculation. Also, others three randomly samples were selected after 21 day after incubation of dentin section blocks with multispecies microorganisms. These samples were inspected to confirm the presence/absence of multispecies bacterial biofilm formation using scanning electron microscope (SEM) as shown in (Figure 1A, B).

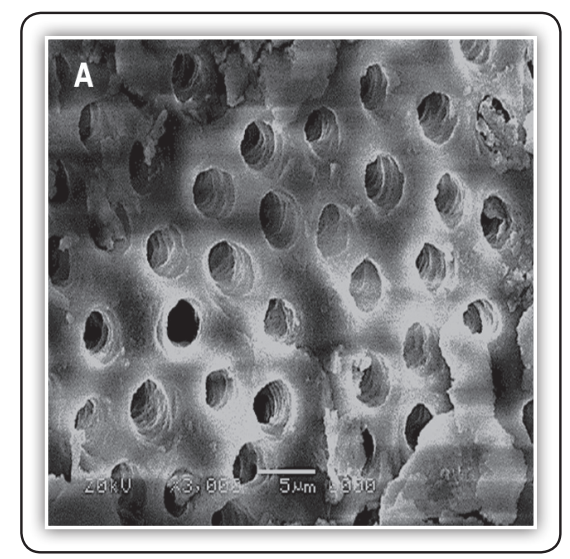

FIG (1-A); Image showing dentin section block immediately after steralization

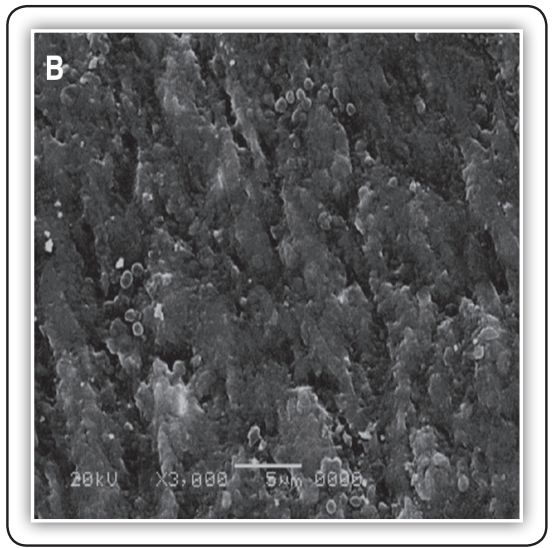

FIG (1-B); Image showing dentin section block after 3 weeks of biofilm inoculation

\section{Preparation of irrigant solutions \& medicaments.}

I- Preparation of pomegranate extract: Pomegranates were obtained from faculty of agriculture Al-Azhar University. The samples then washed, peeled, dried and subsequently powdered. Two hundred grams of powdered pomegranate Peel were equally soaked in three different solvents (Ethanol, ethyl acetate and methanol) at room temperature for 72 hours. Then, the extracts were filtered by Millipore filter then, the filtrated extracts were concentrated in rotary evaporator at $40^{\circ} \mathrm{C}$ then 
the total extracts were tested for antibacterial efficacy using well diffusion agar method taking every solvent as control to determine their antibacterial effect. Samples were stored in a refrigerator at $2-8^{\circ} \mathrm{C}$ for use in subsequent procedures.

Preparation of nano pomegranate extract: The herbal extracts are then turned into nanoherbals by nanoemulsion $\mathrm{O} / \mathrm{W}$ (oil on water) which was done using two-step procedure, Initially coarse emulsion was prepared by mixing the paraffin oil $(1 \mathrm{ml})+$ (1 gram extract) and surfactant Tween $80(2 \mathrm{ml})$ followed by addition of distilled water $(7 \mathrm{ml})$ in the ratio 1:2:7 using magnetic stirrer at $600 \mathrm{rpm}$ for $10 \mathrm{~min}$ at $25 \mathrm{C}$. Then, prepared coarse emulsion was further sonicated using sonicator with a maximum power output of $750 \mathrm{~W}$ under ice bath to avoid rising in temperature. The sonication process was carried out for $10 \mathrm{~min}$.

Characterization of transformation to nanoherbals: Nanoemulsion was characterized by transmission electron microscopy (TEM) at magnification 20000 and it showed nano emulsion spherical in shape with average size of 50 nanometer (Figure 2)

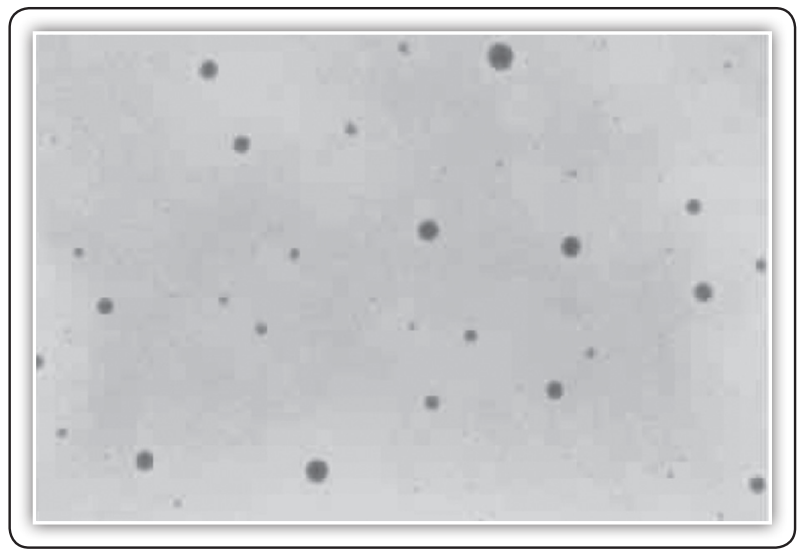

FIG (2) T.E.M. showing nano pomegranate emulsion with spherical shape with average size of 50 nanometer at magnification of 20000x

Preparation of the Nanoherbal gel: Methylcellulose, N,N-dimethylacetamide, 1,2-propylene glycol-1,2, purified water all were used. Methylcel- lulose hydrogels at $3 \%$ concentration and $10 \%$ propylene glycol-1,2 and 5\% N,N-dimethylacetamide distilled water and nanoemulsion were used to produce methyl cellulose nanoherbal gel to produce a base gel containing nanoherbal with concentration of $50 \mathrm{mg} / \mathrm{ml}$.

\section{II- Rose Rose Bengal dye (Photosensitizer} agent): Stock solution of Rose Rose Bengal dye (Sigma-Aldrich, St Louis, MO. USA) was prepared by dissolving $20 \mathrm{mg} \mathrm{RB}$ powder in $20 \mathrm{ml}$ deionized water to obtain a final concentration $10 \mu \mathrm{M} / \mathrm{mL}$ of $\mathrm{RB}$ dye after vortexed for $3 \mathrm{~min}$ at $37^{\circ} \mathrm{C}$. the stock solution of Rose Bengal dye was stored at $4^{\circ} \mathrm{C}$ when not needed.

Characterization of Rose Bengal (RB) dye (Spectrophotometric analysis of RB dye solution): The absorption spectra of diluted solution of the prepared RB dye was recorded within 550 to $750 \mathrm{~nm}$ using double beam UV-Visible Spectrophotometer and the maximum absorption peak was recorded at $650 \mathrm{~nm}$

7. Grouping of the samples: All dentin section blocks were randomly classified into 5 groups ( $n=20), 4$ experimental groups $(n=20)$, and 1 negative control group (None treated bacterial group) ( $n=20)$ According to the type of the final rinse of irrigant and herbal medicament applied.

- Group (1) Medicated with nano punica granatum herbal extract medicament

- Group (2) Medicated with calcium hydroxide paste (Urbical promedica Germany).

- Group (3) Final rinse using Rose Rose Bengal dye activated with diode laser

- Group (4) Final rinse using sodium hypochlorite $5.25 \%$ irrigation.

- Group (5) Non-treated group as negative control group. 


\section{Application of the irrigant solutions and medicament}

8.1. Final rinse protocol: Before application of the final rinse, the dentin section blocks for each group were centrifuged (4,000 rpm, 2 minutes) for allowing the biofilm to precipitate on the dentin section blocks. The samples of each group were opened in a sterile air laminar flow cabinet and the supernatants of the broth media were aspirated with a sterile $3 \mathrm{ml}$ plastic syringe leaving the contaminated dentin section blocks to deliver the final rinse irrigation and medicament except the control group that remain without any treatment until evaluation.

\subsection{Final rinse and medicament application:}

Group (1): Medicated with nano punica granatum herbal extract medicament

Each sample was medicated with $1 \mathrm{ml}$ of nano punica granatum herbal extract medicament within the Eppendorf tube till its fully covered and incubated in the incubator at $37^{\circ} \mathrm{C}$ for 7 days at humidity $100 \%$

Group (2): Medicated with Calcium hydroxide medicament.

Each sample was medicated with $1 \mathrm{ml}$ of Calcium hydroxide medicament (Urbical promedica Germany) within the Eppendorf tube till it is fully covered and incubated in the incubator at $37^{\circ} \mathrm{C}$ for 7 days at humidity $100 \%$

Group (3): Final rinse using Rose Rose Bengal dye $(\mathrm{RB})$ and activated with diode laser (Photodynamic therapy).

Each sample was rinsed with $1 \mathrm{ml}$ of Rose Rose Bengal dye solution $(10 \mu \mathrm{M})$ concentration within the Eppendorf tube and protected from ambient light then left for 15 minutes (prior to irradiation) for allowing the dye to interact with biofilm. Irradiation was performed using a diode laser (Lasotronix device) $635 \mathrm{~nm}$ for 5 minutes at energy fluence $30 \mathrm{~J} / \mathrm{cm} 2$.
Group (4): Final rinse using sodium hypochlorite $(\mathrm{NaOCl})$.

Each sample was rinsed with $1 \mathrm{ml}$ of sodium hypochlorite $(\mathrm{NaOCl})$ solution $5.25 \%$ concentration within the Eppendorf tube then left for 15 minutes.

\section{Group (5): Control group.}

There is no final rinse was used in this group. Each sample was left within the Eppendorf tube without any treatment (Negative control group) until time of evaluation.

\section{Evaluation using confocal laser scanning microscopy}

For the analysis of antimicrobial activity using confocal laser scanning microscopy, the samples were removed from the nanoherbal gel and irrigant solutions then washed for $1 \mathrm{~min}$ by deionized water to remove the remnants of the nanoherbal medicaments and irrigant solutions then stained by (Florocine diacetate FDA and propodium iodid PI) to detect Live/Dead bacteria in the bacterial biofilm and left for 15 min then the samples were removed from the stains solutions, placed on slide, covered by cover slide and examined under confocal microscope. The data were collected, tabulated, and then stastically analyzed.

\section{Statistical analysis}

The mean and standard deviation values were calculated for each group. Viable counts of antibacterial activity were transformed to their $\log 10$ values. Data were confirmed to be normally distributed. All Data were explored for normality using Kolmogorov-Smirnov and Shapiro-Wilk tests. Data showed parametric (normal) distribution. One-way ANOVA followed by Tukey post-hoc test was used to compare between different groups for non-related samples in parametric data. Pairwise sample t-test was used to compare between dependent samples. The significance level was set at $\mathrm{P} \leq 0.05$. Statistical analysis was performed with IBM ${ }^{\circledR}$ SPSS ${ }^{\circledR}$ Statistics Version 20 for Windows. 


\section{RESULTS}

With regard to the antimicrobial effectiveness of tested irrigants and medicaments, the highest mean percentage of dead bacteria was found in nano punica granatum (Group 1) $(88.38 \pm 5.45)$ followed by Rose Bengal dye (RB) (Photodynamic therapy) (Group 3) $(75.73 \pm 5.15)$ followed by sodium hypochlorite $5.25 \%$ (Group 4$)(59.43 \pm 8.14)$ followed by calcium hydroxide paste medicated (Group 2) $(38.73 \pm 5.15)$ . The least mean percentage of dead bacteria was found in Control group (Group 5) (7.25 \pm 1.12$)$. With a statistically significant difference between groups where $(\mathrm{p}<0.001)$ as shown in figure 3 .

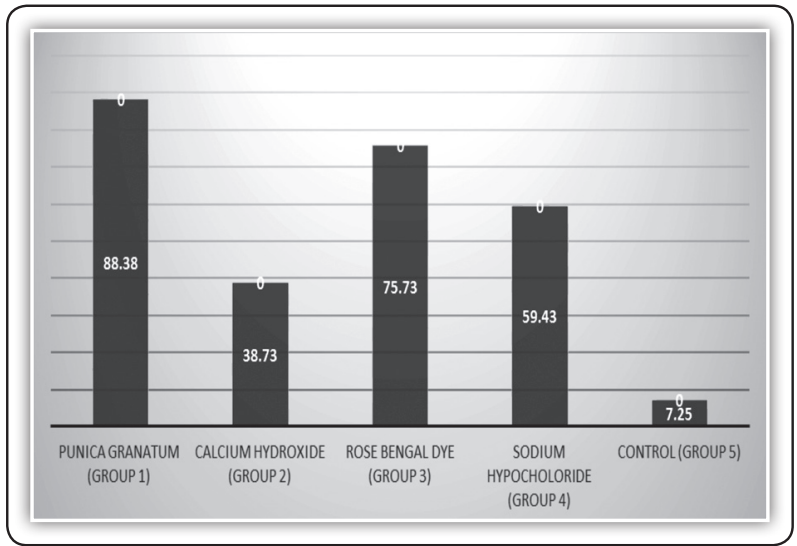

FIG (3) A bar chart representing mean percentage of dead bacteria in control and all experimental groups

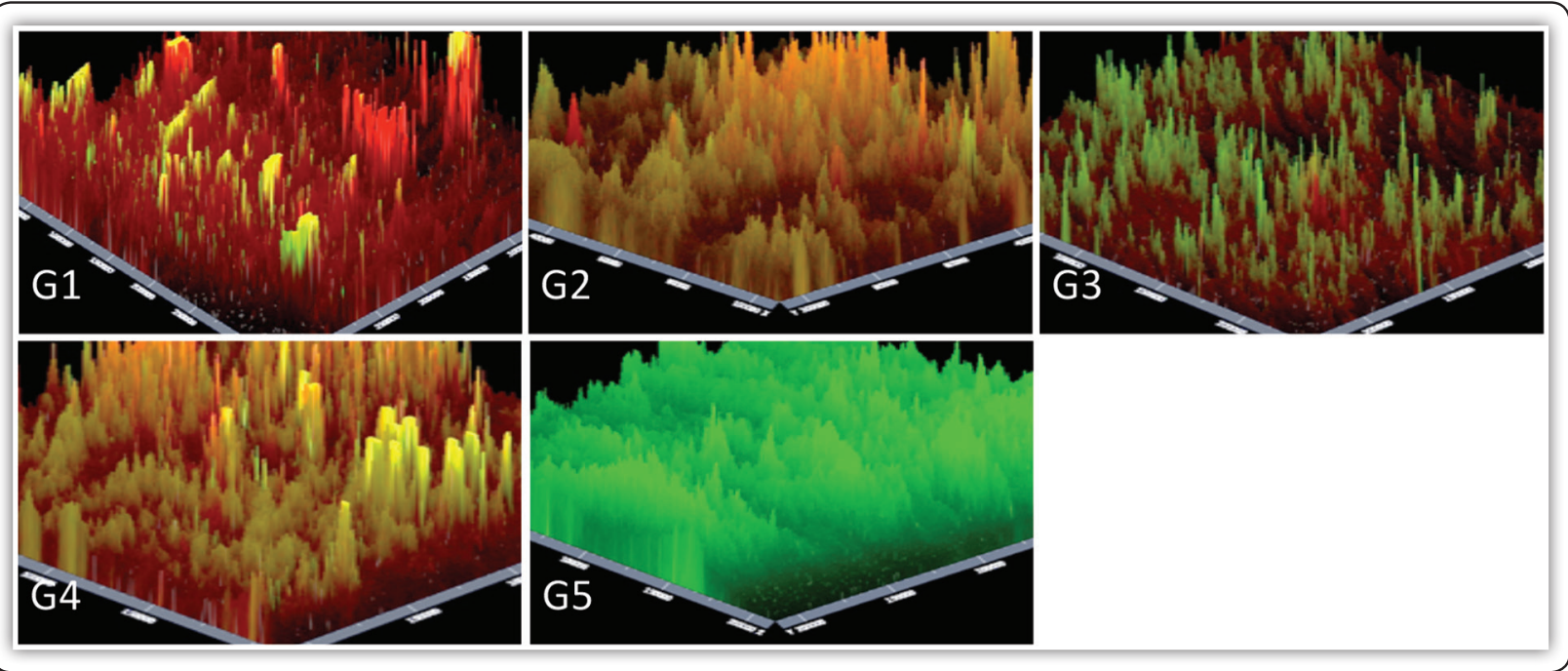

FIG (4) CLSM images for different groups.

Analysis of multispecies biofilm images by CLSM: Figure 4 showing 3D reconstruction of multispecies bacterial biofilm representing live (green) and dead (red) bacteria for different groups

\section{DISCUSSION}

Root canal disinfection is one of the main clinical challenges in endodontic therapy due to the physical limitations of irrigation within a closed system, anatomical root complexities and bacterial accumulation in the forms of bacterial biofilms ${ }^{(16-18)}$ Many methods have been utilized to efficiently disinfect the root canal system including different irrigant solutions with different activation methods $^{(19)}$. This study was directed to evaluate the antibiofilm efficacy of nano-herbal medicament and photodynamic therapy for eradication of multispecies bacterial biofilm within dentin section blocks. One hundred recently extracted single rooted teeth were collected in this study. Single rooted teeth were selected in this study because of its liability to have a single straight root canal in a bulky root that is more suitable for dentin section blocks preparation with preset dimension. The teeth were collected from patients with ages ranging between 20 and 
40 years to minimize variation in root dentin nature that may have effect on lumen size of the root canals ${ }^{(20)}$. The crowns of teeth were cut off to standardize a reference point and to facilitate the instrumentation of canals. Dentin section blocks were used in this study as a biofilms model to closely simulates as possible the in vivo root canal environment ${ }^{(21)}$. The dentin section blocks were prepared with dimensions $(4 \times 4 \times 1 \mathrm{~mm})$ (Length $\times$ Width $\times$ thickness) respectively. These dimensions were standardized to be matched with the modified chamber room device of CLSM for ease of samples evaluation. Also, the laser source of CLSM cannot inspect the samples with dimensions more than $1 \mathrm{~mm}$ in thickness ${ }^{(22)}$. Other researchers ${ }^{(21,23,24)}$ used dentin section blocks having the same parameters. A digital caliper was used to confirm the standardization of all samples size. Any dentin section blocks with dimensions more/less than $(4 \times 4 \times 1 \mathrm{~mm})$ (Length $\times$ Width $\times$ thickness) and lumen size more/less than $1.5 \mathrm{~mm}$ in width were excluded from the study. Each Eppendorf tube containing dentin section block was filled with brain heart infusion (BHI) broth to facilitate confirmation of sterilization of the samples by bacterial count test. The E.faecalis bacteria was selected for this study because it is the most resistance bacteria associated with different forms of periradicular diseases including primary and persistent endodontic infections. Also, it has ability to attach itself to dentin wall and invade dentinal tubules forming biofilm communities able to resist irrigation solutions and intracanal medicaments ${ }^{(25)}$. Staphylococcus epidermidis was selected because it had been associated with endodontic infection, and for synergistic relationship with E.faecalis to form a multispecies biofilm ${ }^{(26)}$. Multispecies biofilm model was selected in this study to closely mimic in vivo biofilm where more than one type of bacteria was isolated from root canal infection $^{(21)}$. Using planktonic cell bacteria or monospecies biofilm doesn't actually reflect the endodontic infection conditions as microorganisms are more likely to be organized in structures attached to each other and/or the root canal walls in form of a multispecies biofilms that make the bacteria 1000-1500 times greater resistant to phagocytosis, antibodies and antimicrobial agents than planktonic state bacteria ${ }^{(27,28)}$. Inoculation of the dentin section blocks with multispecies bacteria was done in a sterile air laminar flow cabinet for assurance of sterilization process, then followed by incubation period 3 weeks to ensure the maturation of multispecies biofilm formation and deeply penetration into dentinal tubules where; the level of biofilm maturation is an essential factor for biofilm resistance to antimicrobial agents (29). For the biofilm development; the bacterial suspension was changed every 3 days to avoid the bacterial endotoxins accumulation that might have led to bacterial death. This allowed the bacterial multiplying without being dampened by their byproducts (30). Dentin section blocks were randomly selected before and after bacterial inoculation then inspected by SEM to confirm the formation of mulispecies biofilm. Pomegranate was used as antibiofilm herbal medicament because of its high contents of hydrolyzable tannins (punicalins and punicalagins), ellagic acid, a component of ellagitannins, and gallic acid, a component of gallotannins. Mass spectrometry data shows that pomegranate contains oligomeric ellagitannin with a degree of polymerization of up to 5 core glucose units. These molecules may be the most potent antibacterial compounds in pomegranate. However, other compounds also have activity and may contribute synergistically as mixtures to bring about the effects, including anthocyanins (pelargonidin-3-galactose and cyanidin-3-glucose) and flavonols (quercetin and myricetin) $)^{(31)}$. Although many intracanal medicaments were introduced to be used to disinfect root canal, calcium hydroxide remains the most wildly used as it exerts antibacterial effects in the root canal system as long as a high $\mathrm{pH}$ is maintained ${ }^{(32)}$ The Rose Bengal dye (RB) was selected as a photosensitizer agent for Photodynamic therapy (PDT) due to its antibacterial activity against various gram positive and gram negative bacteria ${ }^{(33,34 \& 35)}$. Also, RB dye is 
an anionic PS that has been shown excellent binding with cationic polymers such as chitosan nanoparticles. The concentration of anionic RB dye that used in the study was $10 \mu \mathrm{M}$. At this concentration, the singlet oxygen that produced was sufficient to induce significant biological activity against bacterial biofilm and this concentration also was founded to be below the cytotoxic limit based on the Shrestha \& Kishen ${ }^{(24)}$ study. Confocal Laser Scanning Microscope (CLSM) was used in this study because of its ability to determine the microbial biofilms at different levels of the infected root canal. Also, it provides detailed information about the presence, distribution, viability status, and depth of penetration of biofilm inside dentinal tubules in accurate 3D reconstructions images. The viability status of bacterial biofilm (live/dead bacteria) was determined throughout use of fluorescence-based live/dead staining technique. This technique was performed with two fluorescent dyes that was allowed two color discrimination of the population of living cells from the dead cells population (green/red colored). FDA and PI stains were used to stain live cells and dead cells, respectively ${ }^{(36)}$. FDA is a non-fluorescent permeable dye. When cross the cellular membrane of living cell convert into fluorescein dye (green in color) by interaction with intracellular Esterases (produced by metabolic activity of microorganisms). PI is a fluorescent impermeable dye that cannot cross the cellular membrane of living cells. It reaches the nucleus by passing throughout disordered areas of dead cell membranes and staining the nuclei (red in color) by intercalate with the DNA double helix of the cell. Thus, live bacterial cells are fluorescent green, whereas dead bacteria with damaged membranes are fluorescent red in color ${ }^{(37)}$. In the present study, the control group (group 5) showed the least mean percentage of dead bacteria when compared to other groups. The percentage of dead bacteria was found to be $7.25 \%$ which is the normal for any untreated bacterial population. This result is in agreements with other researches done in this field ${ }^{(38,39)}$. This can be explained by the fact of the absence of disinfecting agent in the control group that has lethal effect on the multispecies bacterial biofilm. With regard to pomegranate nanoherbal (group 1) it showed the highest significant absolute mean score of dead bacterial cells and these results were coincided with results reported by shoko T. et al. ${ }^{(40)}$ these findings may be attributed to that pomegranate extracts contains phenolic, anthocyanin, flavonols and organic acids. In accordance to our study the antimicrobial properties of $\mathrm{P}$. granatum have been recently noticed ${ }^{(41)}$. The ethanol, water, methanol, and acetone extracts of P. granatum have shown strong antimicrobial properties against gram-positive and gram negative nonoral microorganisms ${ }^{(42)}$. However, A few studies have evaluated the antibacterial properties of this plant on oral bacteria ${ }^{(43)}$. The effect of water extract of $\mathrm{P}$. granatum flower (petal) on oral microbial pathogens was investigated, which showed its greatest antimicrobial effect on S. sanguinis. Also, in accordance to the present study, Gulube and Patel ${ }^{(44)}$ investigated pomegranate on biofilm formation, the crude extract of pomegranate killed cariogenic S. mutans at high concentrations. At sub-bactericidal concentrations, it reduced biofilm formation. Irshad R. et al. ${ }^{(45)}$ revealed that pomegranate nanoparticles are found to have high antibacterial activity along with biocompatibility. This high antibacterial activity can be referred to its small size and large surface area. With regard to calcium hydroxide medicament (group 2) the multispecies biofilms on dentin section blocks were destroyed after 7 days of treatment, whereas several live bacteria still remained on the dentin sections. Possibly the high alkalinity of calcium hydroxide was neutralized by the dentin and biofilm matrix of multispecies biofilms ${ }^{(46,47)}$ attributing to the reduced antibacterial effect of calcium hydroxide. With regard to RB dye group (group 3), The result of dead bacteria could be attributed to the fact of $\mathrm{RB}$ dye is an anionic photosensitizer (negatively charged photosensitizer) that does not interact electrostatically with the negatively charged bacterial cell membranes, resulting in the membrane barriers of the bacterial cells limit the simple diffusion of 
RB dye into the bacteria cells. This leaded to reduced penetration of the Rose Bengal dye in to bacterial biofilm within dentinal tubules ${ }^{(48)}$. Aso, these results are in agreements with other researches done in this field ${ }^{(49,50)}$. With regard to sodium hypochlorite group (group 4), the result of dead bacteria could be attributed to the fact of sodium hypochlorite in concentration $5.25 \%$ demonstrated antibiofilm effect against $E$. faecalis biofilm. The proportion of live bacteria in the $5.25 \%$ sodium hypochlorite group could be measured by viability staining and CLSM because sodium hypochlorite destroyed E. faecalis biofilm so quickly, leaving very little residual biofilm on dentin sections for analysis ${ }^{(51)}$. Time of tested materials application was done in accordance to clinical resemblance and it is expected to affect the results of the present study

\section{CONCLUSION}

Nano punica granatum herbal extract medicament (gel/cream) and Rose Bengal dye (RB) irrigation (Photodynamic therapy) could be considered potent antibiofilm strategies for disinfection of the root canal system.

\section{REFERENCES}

1. Rocas I, Siqueira J. Identification of bacteria enduring endodontic treatment procedures by a combined reverse transcriptase-polymerase chain reaction and re verse-capture checkerboard approach. J Endod 2010; 36:45-52.

2. Torabinejad M, Handysides R, Khademi A, Bakland L. Clinical implications of the smear layer in endodontics: a review. Oral Surg Oral Med Oral Pathol Oral Radiol Endod 2002; 94:658-66.

3. Kayaoglu G, Ørstavik D. Virulence factors of Enterococcus faecalis: relationship to endodontic disease. Crit Rev Oral Biol Med 2004; 15: 308-20.

4. Stewart P, Costerton J. Antibiotic resistance of bacteria in biofilms. Lancet 2001; 358:135-8.

5. Waltimo T, Trope M, Haapasalo M, Ørstavik D. Clinical efficacy of treatment procedures in endodontic infection control and one year follow-up of periapical healing. J En$\operatorname{dod} 2005 ; 31: 863-6$.
6. Peters O, Laib A, Gohring T, Barbakow F. Changes in root canal geometry after preparation assessed by high-resolution computed tomography. J Endod 2001; 27:1-6.

7. Estrela C, Estrela CR, Barbin EL, et al. Mechanism of action of sodium hypochlorite. Braz Dent J 2002; 13:113-7.

8. Lee M, Winkler J, Hartwell G, et al. Current trends in endodontic practice: emergency treatments and technological armamentarium. J Endod 2009; 35:35-9.

9. Siqueira JF Jr, Lopes HP. Mechanisms of antimicrobial activity of calcium hydroxide: a critical review. Int Endod J 1999; 32:361-9.

10. Haapasalo HK, Siren EK, Waltimo TM, et al. Inactivation of local root canal medicaments by dentine: an in vitro study. Int Endod J 2000; 33:126-31.

11. Hamblin M, Hasan T. Photodynamic therapy: a new antimicrobial approach to infectious disease. Photochem Photobiol Sci 2004; 30: 436-50.

12. George $\mathrm{S}$, Kishen A. Influence of photosensitizer solvent on the mechanisms of photoactivated killing of Enterococcus faecalis. Photochem Photobiol 2008; 84: 734-40.

13. Cushing B, Kolesnichenko V, O'Connor C. Recent advances in the liquid-phase syntheses of inorganic nanoparticles. Chem Rev 2004; 104: 3893-946.

14. Farmahan, H. "Pomegranate," in Recent Trends in Horticulturein the Himalayas, NewDehli, India, 2004.

15. Jayaprakasha G. Negi, and B. Jena, "Antimicrobialactivities of pomegranate," in Pomegranates: Ancient Roots to Modern Medicine, 168, CRC Press, New York, NY, USA, 2006.

16. Haapasalo M, Ørstavik D. In vitro infection and disinfection of dentinal tubules. J Dent Res 1987; 66:1375-9.

17. Gregorio C, Arias A, Navarrete N, Rio V, Oltra E, Cohenca N. Effect of Apical Size and Taper on Volume of Irrigant Delivered at Working Length with Apical Negative Pressure at Different Root Curvatures. J Endod 2013; 39:119-24.

18. Tay C, Quah S, Lui J, Yu V, Tan K. Matrix Metalloproteinase Inhibitor as an Antimicrobial Agent to Eradicate Enterococcus faecalis Biofilm. J Endod 2015; 41:1-6.

19. Kishen A. Advanced therapeutic options for endodontic biofilms. Endod Topics 2010; 22:99-123.

20. Morse D, Age-related changes of the dental pulp complex and their relationship to systemic aging. Oral Surg Oral Med Oral Pathol. 1991; 72:721-45 
